\title{
Tuning of Spinal Networks to Frequency Components of Spike Trains in Individual Afferents
}

\author{
H. Richard Koerber, Andrew W. Seymour, a and Lorne M. Mendell \\ Department of Neurobiology and Behavior, SUNY at Stony Brook, Stony Brook, New York 11794
}

Cord dorsum potentials (CDPs) evoked by primary afferent fiber stimulation reflect the response of postsynaptic dorsal horn neurons. The properties of these CDPs have been shown to vary in accordance with the type of primary afferent fiber stimulated. The purpose of the present study was to determine the relationships between frequency modulation of the afferent input trains, the amplitude modulation of the evoked CDPs, and the type of primary afferent stimulated. The somata of individual primary afferent fibers were impaled in the L7 dorsal root ganglion of $\alpha$-chloralose-anesthetized cats. Action potentials (APs) were evoked in single identified afferents via the intracellular microelectrode while simultaneously recording the response of dorsal horn neurons as CDPs, or activity of individual target interneurons recorded extracellularly or intracellularly. APs were evoked in afferents using temporal patterns identical to the responses of selected afferents to natural stimulation of their receptive fields. Two such physiologically realistic trains, one recorded from a hair follicle and the other from a slowly adapting type 1 receptor, were chosen as standard test trains. Modulation of CDP amplitude in response to this frequency-modulated afferent activity varied according to the type of peripheral mechanoreceptor innervated. Dorsal horn networks driven by $A_{\theta}$ afferents innervating hair follicles, rapidly adapting pad (Krause end bulb), and field receptors seemed "tuned" to amplify the onset of activity in single afferents. Networks driven by afferents innervating down hair follicles and pacinian corpuscles required more high-frequency activity to elicit their peak response. Dorsal horn networks driven by afferents innervating slowly adapting receptors including high-threshold mechanoreceptors exhibited some sensitivity to the instantaneous frequency, but In general they reproduced the activity in the afferent fiber much more faithfully. Responses of synaptically coupled dorsal horn neurons belonging to either hair follicle or SA1 fiber-driven networks to frequency-modulated input were in agreement with the CDP results, confirming that CDP amplitude mod-

\footnotetext{
Received Feb. 20, 1991; revised May 13, 1991; accepted May 22, 1991

We thank Drs. Eric Frank and Paul Brown for useful comments on the manuscript and Dr. Brown for his participation in the initial experiment of this series. Dr. Nancy Mendell of the Department of Applied Mathematics and Statistics, SUNY at Stony Brook aided with statistical evaluations. This work was supported by NIH Grants ROI NS23275 (H.R.K.), RO1 NS16996 (L.M.M.), and PO1 NS14899 (L.M.M.).

Correspondence should be addressed to H. Richard Koerber, Department of Neurobiology, Anatomy and Cell Science, University of Pittsburgh School of Medicine, Pittsburgh, PA 15261.

Present address: Department of Pathology, Kings County Hospital, 451 Clarkson Avenue, Brooklyn, NY 11203.

Copyright (c) 1991 Society for Neuroscience $0270-6474 / 91 / 113178-10 \$ 05.00 / 0$
}

ulation is a true reflection of EPSP amplitude modulation in at least a subset of dorsal horn neurons comprising the network.

Processing of cutaneous information in the dorsal horn has been investigated recently by evoking single action potentials (APs) and pairs of APs in individual primary afferents and recording the responses of synaptically coupled dorsal horn neurons (Brown et al., 1973; Tapper and Wiesenfeld, 1980; Tapper et al., 1983, 1985; Craig and Tapper, 1985; Brown et al., 1987a-c). These studies were concerned primarily with transmission of information from two different afferent types, hair follicle and slowly adapting type 1 (SA1) afferents. Taken together, these studies suggested little variation in the response of dorsal horn neurons to individual APs in these different afferent types. However, some differences among afferents innervating these different receptor types could be seen in the response to pairs of APs.

More recently, the functional projection of many different identified afferent types has been studied using cord dorsum potentials (CDPs) evoked by single fiber stimulation (Koerber and Mendell, 1988; Koerber et al., 1990). These potentials measure the summed synaptic activity of the neurons activated by the peripheral stimulation (reviewed in Willis and Coggeshall, 1978). The results from these studies, using various frequencies of single AP stimulation as well as pairs of APs, suggested that both the monosynaptic response and that of polyneuronal dorsal horn networks activated by single afferents vary according to the type of peripheral mechanoreceptor supplied by the afferent (Koerber and Mendell, 1988; Koerber et al., 1990).

Despite these findings, it is important to recognize that under normal conditions the inputs arriving in these afferent fibers are in the form of relatively short, frequency-modulated bursts of activity rather than the single APs or pairs of APs used previously to investigate transmission in the spinal cord. In order to explore more fully the input-output relations of the afferent/ spinal network projections, it is necessary to subject the afferent fibers to these physiologically realistic patterns of input (e.g., Collins et al., 1986; Koerber and Mendell, 1991). Thus, the present series of experiments was performed in order to examine the response of dorsal horn networks to physiologically realistic inpul trains that were derived from the response of primary afferent fibers to natural stimulation of their receptors.

A preliminary report of this study has been previously published (Koerber et al., 1989).

\section{Materials and Methods}

Adult mixed strain cats of either sex were preanesthetized with a mixture of ketamine (Ketoset, $20 \mathrm{mg} / \mathrm{kg}$ ) and xylazine (Rompun, $0.5 \mathrm{mg} / \mathrm{kg}$ ). A carotid artery and jugular vein were cannulated to allow monitoring of 
blood pressure and infusion of drugs. The anesthetic was then switched to $\alpha$-chloralose $(70 \mathrm{mg} / \mathrm{kg})$ by intravenous infusion. The trachea was cannulated, and the animal was paralyzed with gallamine triethiodide (Flaxedil). The animals were ventilated, and end-tidal $\mathrm{CO}_{2}$ was monitored and maintained between $3.5 \%$ and $4.5 \%$. A laminectomy was performed to expose the lumbosacral enlargement and the L7 dorsal root ganglion, and the sciatic nerve of the left hindlimb was dissected free in the popliteal fossa and placed on platinum hook electrodes for stimulation. The dura was cut, and the spinal cord was covered with warm $\left(37^{\circ} \mathrm{C}\right)$ mineral oil. Four bipolar metal electrodes (30 gauge platinum) were positioned over the spinal cord, with the active lead in contact with the surface of the spinal cord and the indifferent lead placed in the surrounding muscle (Fig. $1 A$ ). The location of each electrode was determined using criteria described in Koerber and Mendell (1988).

The L7 dorsal root ganglion (DRG) was freed from surrounding fascia and placed on a metal platform. The epineural sheath surrounding the ganglion was slit with a microknife to permit microelectrode penetration of the ganglion. Sciatic nerve stimulation $(2 \mathrm{~Hz})$ served as a search stimulus as a microelectrode was advanced through the ganglion in search of responding units. After impalement of a cell, its peripheral receptor was identified (Horch et al., 1977) and the conduction delay recorded. The cell was then stimulated via the intracellular electrode with single brief pulses $(<0.2 \mathrm{msec})$ to produce single action potentials (APs) or trains of APs while simultaneously averaging the resulting cord dorsum potentials (CDPs) in register (see Collins et al., 1984). In all cases, the reliability of stimulation was verified by monitoring the impulse produced by the intracellular stimulation. Averaged CDPs were stored on magnetic tape for later analysis using a Nicolet 1170 averager. Thus, the response to each pattern of stimulation in each afferent was recorded at four positions; the largest of the four responses was analyzed.

Frequency-modulated trains. The frequency-modulated trains used in this study were derived from the response of individual afferents to natural stimulation of their peripheral receptor. Two spike trains were selected as standard test stimuli and were delivered with an interburst interval of $2 \mathrm{sec}$. These trains were chosen because they were realistic, and no exhaustive attempt was made to ensure that they were representative. The first (train 1) was recorded from an afferent innervating a guard hair follicle in response to a brief puff of air applied to its receptive field. This spike train consisted of six action potentials with the following interspike intervals: $3.0,10.1,3.2,22.9$, and $7.7 \mathrm{msec}$ (Fig. 2). The second train (train 2) was recorded from a slowly adapting type 1 (SA1) mechanoreceptor in response to a stroke across the receptive field using a small, blunt wooden probe. This train consisted of nine action potentials with interspike intervals of 15.7, 14.6, 19.9, 13.6, $7.5,25.7,5.6$, and $3.3 \mathrm{msec}$ (Fig. 2). (Note that the intervals between the first two spikes of train 1 and the last two spikes in train 2 are roughly equal.) These intervals were stored and reproduced on demand using a programmable stimulus control system (SLC-1, Stoelting). This allowed the reproduction of these standard trains in other single afferents via the intracellular microelectrode.

Paired single-unit recordings. In three experiments, activity was recorded from single interneurons activated by the impaled cutaneous afferents to verify that modulation of CDP amplitude reflected changes in the synaptic drive in individual spinal neurons. Following placement of the cord dorsum electrodes and impalement of an identified afferent in the DRG, the afferent was stimulated at high frequency and CDPs recorded. This verified that the central projections of the impaled afferent were functional and identified the rostrocaudal location of the peak CDP amplitude response and therefore peak density of that fiber's boutons (Koerber et al., 1990). This located the rostrocaudal level at which there would be a high probability of finding synaptically coupled dorsal horn neurons.

Micropipettes filled with $3 \mathrm{~m}$ potassium acetate were used to search for dorsal horn neurons responding to either sciatic nerve stimulation or manual stimulation of the skin (Fig. $1 B$ ). Once a cell was isolated, its response properties [e.g., low threshold only, or wide dynamic range (WDR)] were determined and its receptive field was mapped. If the dorsal horn ccll's reccptive ficld contained that of the impaled DRG cell, the afferent was stimulated via the intracellular electrode and the dorsal horn cell's response was recorded on videotape for off-line analysis (i.e., peristimulus time histograms). Following these procedures, an attempt was made to penetrate the dorsal horn neuron. If a successful penetration was achieved ( $|R M P|>60 \mathrm{mV}$ ), the cell's receptive field was remapped and the single afferent stimulus patterns repeated. The intracellular responses were recorded on tape for later analysis.

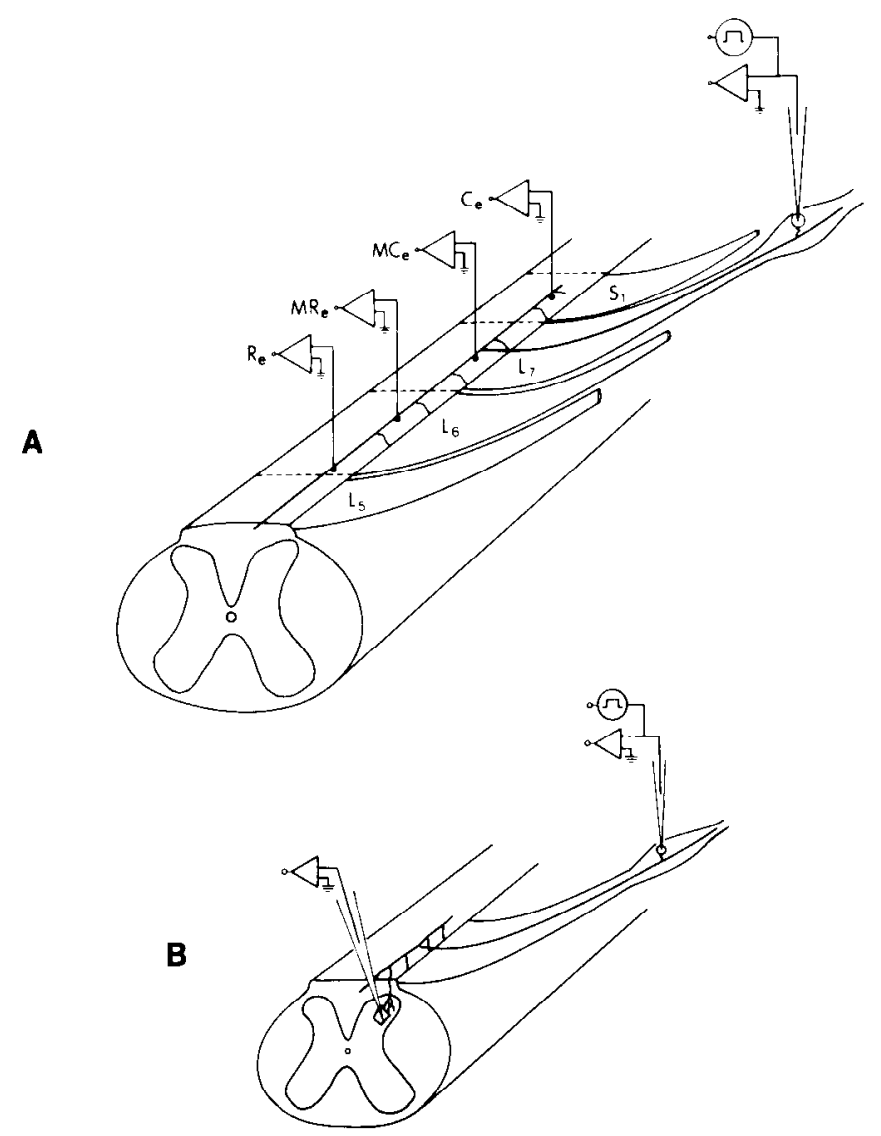

Figure 1. A, Schematic diagram of electrode arrangement for recording CDPs. Brief depolarizing current pulses passed through microelectrode in DRG cell evoke individual APs in the sensory neuron, and the resultant CDPs are recorded simultaneously by the four monopolar electrodes placed on the surface of the spinal cord. Indifferent electrodes were placed on adjacent muscle. $B$, Arrangement for recording from target interneurons. Spinal cord electrode can be either intracellular, as shown, or extracellular.

\section{Results}

Seventy-eight afferent fibers were impaled, characterized, and stimulated in seven experiments in which only single-fiber CDPs were recorded. Of those 78 fibers, 26 were characterized as innervating hair follicles (guard or tylotrich); 9 , field receptors; 5 , pacinian corpuscles (PCs); 5, rapidly adapting (RA) pad (Krause end bulb) receptors; 8 , down hair (D-hair) receptors; 15 , SAls (in both hairy and glabrous skin); 4, SA2s; and 6, high-threshold mechanoreceptors (HTMRs). In an additional three experiments, the responses of individual dorsal horn cells (DHCs) to stimulation of identified primary afferents (three $A_{\beta}$ hair follicle afferent fiber-DHC pairs and three SAl-DHC pairs) were recorded.

\section{Cord dorsum potentials}

The CDP averaged in response to the repeated short bursts of frequency-modulated activity in single fibers varied according to the cutaneous receptor type supplied by the stimulated afferent. An example of this can be seen in Figure 2, where an SA1 afferent was stimulated using three different paradigms. First, the monosynaptic CDP was recorded in response to continuous $18 \mathrm{~Hz}$ stimulation of the fiber (1024 trials) with results that were identical to those reported previously (Koerber and 


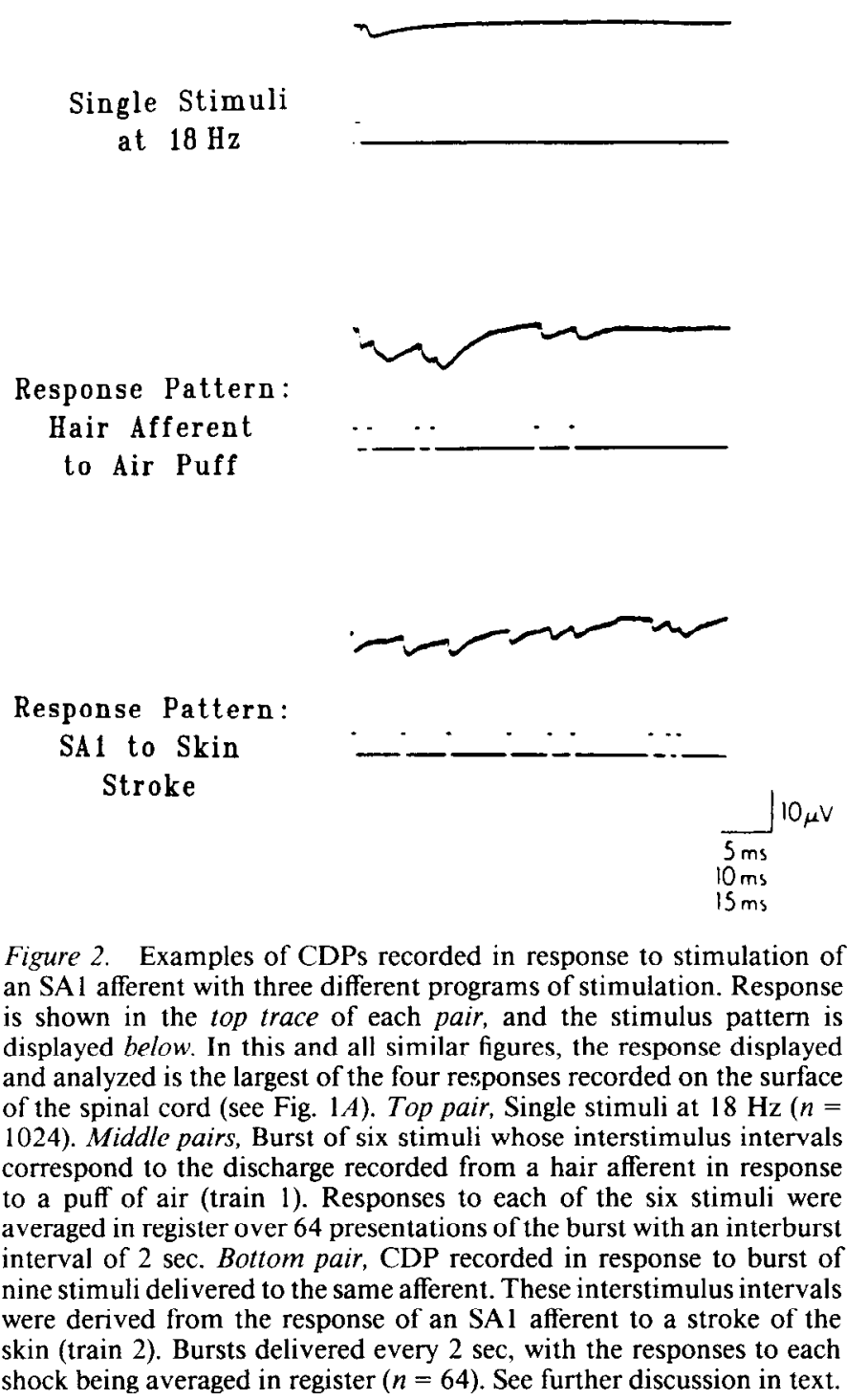

Mendell, 1988; Koerber et al., 1990). This figure also displays examples of the averaged CDPs $(n=64)$ (all CDPs are the average response to 64 presentations unless stated otherwise) evoked by the two standard frequency modulated spike trains, train 1 (hair afferent response to air puff) and train 2 (SA1 afferent response to stroking the skin). Stimulation of this afferent fiber (SA1) evoked resolvable negative CDPs in response to individual stimuli in each train (displayed beneath the CDP traces). However, when these same trains were "played" into other types of cutaneous mechanoreceptors, the resulting CDPs were quite different in appearance.

The amplitude modulation of CDPs evoked in response to stimulation of six different types of single afferent fibers using train 1 fell into three general categories (Fig. 3). The first such group is represented by the responses to stimulation of the two fibers supplying SA receptors, an SA1 and an HTMR (top two traces). Although the amplitude of these responses could exhibit some sensitivity to instantaneous frequency (i.e., amplification of the second of two responses in a high-frequency pair delivered to the SAl afferent), these networks responded quite faithfully to each stimulus in the input train in comparison to the other groups. The second group consisted of networks driven by $A_{\beta}$ afferents supplying certain rapidly adapting receptors [i.e., hair,

\section{TRAIN 1}

SA 1

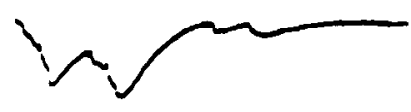

$A_{\beta}-\mathrm{HTMR}(\mathrm{WDR})$

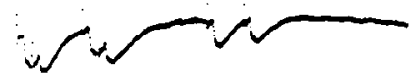

Hair

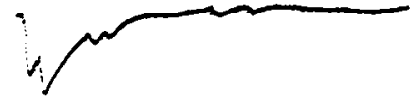

RA-pad

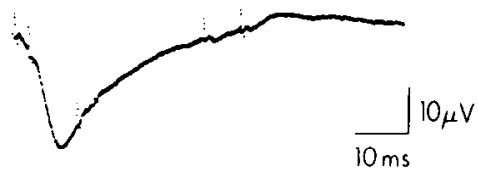

Pac. Corp.

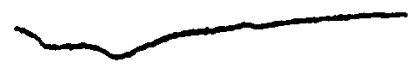

D-Hair

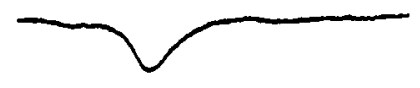

Response Pattern:
Hair Afferent
to Air Puff

Figure 3. CDP patterns in response to "air puff" pattern (train 1): six afferents innervating different receptor types. See further discussion in text. Pac. Corp., pacinian corpuscle.

field (not shown), and RA pad]. These networks (Fig. 3, center two traces) responded best to the first pair of stimuli in the train and thus appeared to be "tuned" to the onset of activity in their afferents. Networks driven by afferents supplying other types of RA receptors ( $\mathrm{PC}$ and $\mathrm{D}$-hair receptors) responded in a third way to this train. Although there was initial facilitation of the network response followed by apparent depression, these afferent fiber-driven networks were "tuned" such that they required a greater number of high-frequency stimuli before they became fully responsive, that is, they only began to respond optimally after the third and fourth stimuli.

In order to compare the CDPs evoked by stimulation of many different afferent types directly, the temporal characteristics of train 1 were considered. This train consists of three pairs of closely spaced spikes that evoked CDPs that could not be resolved individually. Therefore, as an estimate of the modulation of synaptic activity during the burst of afferent activity, we compared the peak amplitude of the CDPs evoked in response to the first (P1), second (P2), and third (P3) pairs of stimuli.

Our use of amplitude rather than area was considered acceptable because we were comparing the ratios of the components produced by stimulation of individual afferents rather than the magnitude of any portion of the response. The ratio of amplitudes was considered to be a reasonable estimate of the ratio of the areas. We made no correction for the fact that the 


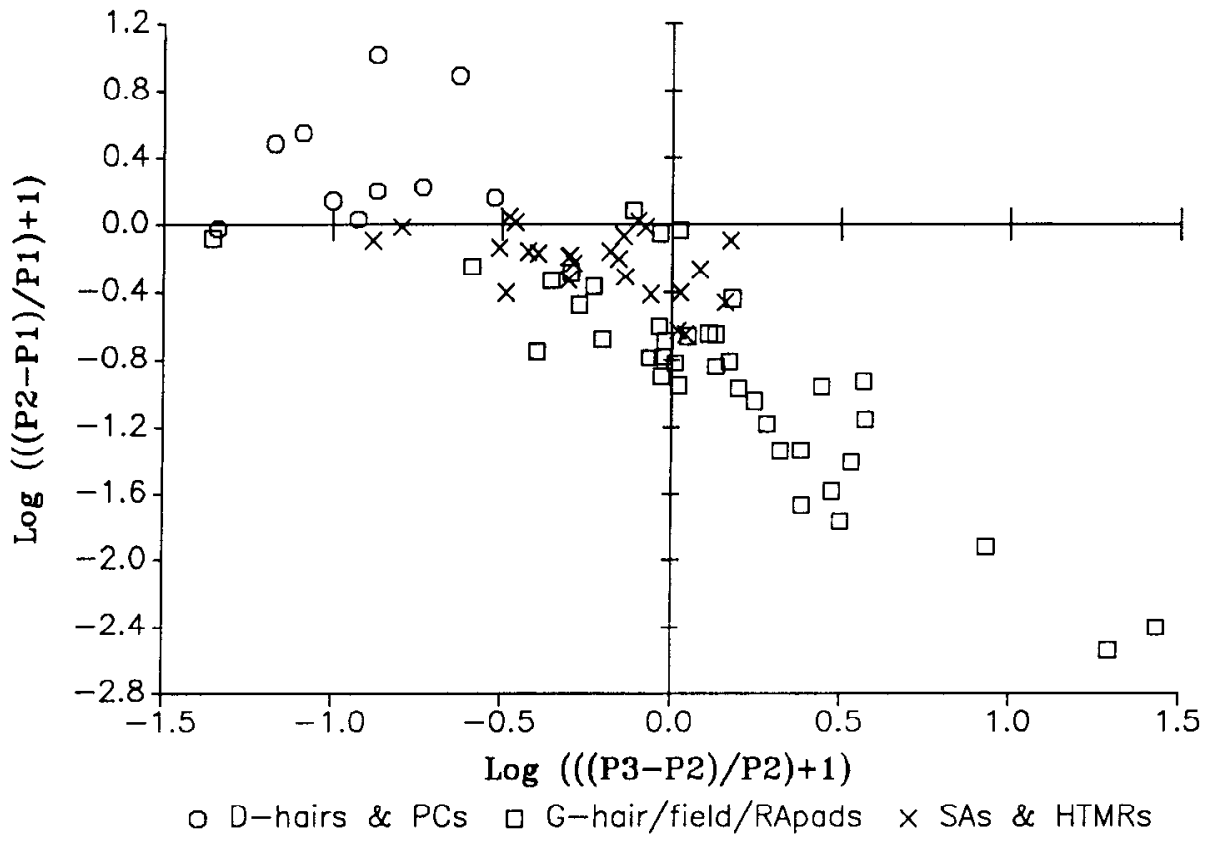

Figure 4. Plot of log of fractional difference between the amplitude of the peak responses to the first and second pair of stimuli $(\mathrm{P} 2-\mathrm{P} 1 / \mathrm{P} 1)$ in train 1 and the $\log$ of the fractional difference between the amplitude of the peak responses to the second and third pair of stimuli (P3 - P2/P2). The quantity "1" is added to each ratio to avoid negative ratios for which $\log$ is not defined. Note that the points associated with different receptor groupings tend to segregate in different portions of this graph. See further discussion in the text. second and third responses were often superimposed on a sloping baseline (Fig. 3). This would have required numerous control measurements to be made (i.e., first pair alone, first and second pairs alone), which was not practical under the conditions of these experiments. Although this caused our determination of these amplitude ratios to be somewhat in error, the systematic variability in these ratios for the different afferent types (Fig. 4) matches the qualitative assessment reached from inspection of records such as those in Figure 3.

The responses of 74 single afferent fiber-driven networks to train 1 stimulation is represented graphically in Figure 4. To demonstrate the different response properties of these networks, the log of the fractional difference between the response to the first (P1) and second (P2) pairs of stimuli was plotted as a function of the $\log$ of the fractional difference between the response to the second (P2) and third (P3) pairs. ANOVA indicated that the three groups of afferents differed in each of the two variables plotted in Figure $4(p<0.01)$. This suggests differential tuning of their spinal networks. For example, data from single fiberdriven networks responding well to the first pair of pulses but not to successive pairs of similar interstimulus intervals (e.g., hair follicle and RA-pad afferents; see Fig. 3) fell mainly in the lower right quadrant (and occasionally in the lower left), whereas points located in the upper left quadrant represent networks that were "tuned" to respond best to the second pair of pulses or possibly to summation of the first four (i.e., PCs and D-hair afferents; see Fig. 3). The SA affercnts gave responscs that wcre roughly the same to each of the three pairs of stimuli (see Fig. 3 ), and these fell closest to the origin.

The same six afferents whose CDPs evoked by train 1 are shown in Figure 3 were also stimulated with a train of pulses corresponding to train 2, with the responses shown in Figure 5. Once again, each impulse discharged by one of the SA afferent fiber types studied (SA1, SA2, and HTMR) evoked CDPs whose amplitudes were relatively uniform. The first group of RA afferent fiber-driven networks (hair, RA pad, and field afferents) again best signaled the onset of the spike train, while exhibiting much less of a response to the later components. The other two types of RA afferent fibers examined (PC and D-hair) did not respond well to any component of this spike train.

As noted above, the intervals between the first two spikes in train 1 and the last two in train 2 are very similar. In addition, the third to last pulse in train 2 precedes the last pair by only $5 \mathrm{msec}$ and could serve to induce additional temporal summation. The same PC and D-hair afferent fiber-driven networks that responded well to the initial four APs of train 1 did not respond well (if at all) to these multiple high-frequency inputs when they occurred at the end of the spike train. Therefore, it appears that these networks are not tuned solely to instantaneous frequency, in that their response properties are sensitive to prior input even if it produces only minimal activity in cells contributing to the CDP (see Collins et al., 1986; Noble and Short, 1989).

The responses of 27 networks driven by train 2 stimulation of SA and RA afferents are displayed graphically in Figure 6. To display the difference in the response properties of RA and SA afferent fiber-driven networks, the fractional difference between the last and initial peak amplitude of the evoked CDPs was plotted against conduction velocity of the axon with different symbols for the various afferent fiber-receptor groupings. RA-driven networks generally exhibited a much greater decline in their response amplitude than SA-driven networks which could exhibit some facilitation. These properties were not correlated with the conduction velocity of the afferent. In some cases, stimulation of RA afferents with this pattern failed to evoke a measurable response (e.g., PC and D-hair afferents; Fig. 5).

\section{Synaptically coupled pairs}

To determine whether the amplitude modulation of the CDPs accurately reflected the amplitude modulation of EPSPs and/or activity in single DHCs, synaptically coupled primary afferent fiber-DHC pairs were recorded simultaneously. The afferent fiber was stimulated while the response of the postsynaptic DHC was recorded as extracellular spike activity or as intracellular EPSPs and APs. 


\section{TRAIN 2}

SA 1

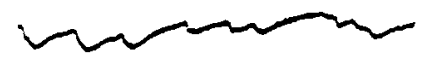

$A_{\beta}-\operatorname{HTMR}($ WDR )

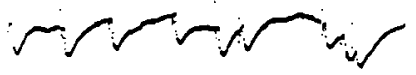

Hair

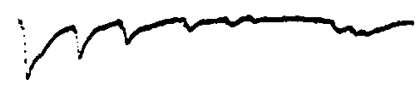

$\mathrm{RA}-\mathrm{pad}$

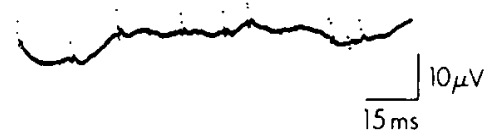

Pac. Corp.

D-Hair

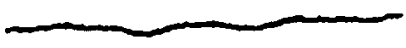

Response Pattern:

SA1 to Skin Stroke

Figure 5. Modulation of CDP amplitude in response to "skin stroke" pattern (train 2) (same afferents as in Fig. 3). See further discussion in text.

\section{Extracellular recordings}

The response of individual DHCs to single afferent fiber stimulation was also observed to vary according to the type of afferent being activated. Examples of this diversity can be seen in Figure 7, where the $\mathrm{DHC}$ responses are displayed as peristimulus time histograms. Figure $7 A$ depicts the response of a dorsal horn neuron classified as WDR (Mendell, 1966) to train 1 delivered to a hair follicle afferent fiber. The synaptic coupling between this pair was very secure $(100 \%$; i.e., the dorsal horn cell responded to every stimulus presentation). Although each train evoked a minimum of two APs in the DHC [mean - 3.1 \pm 1.1 (SD)], it was activated predominantly by the first two APs in the spike train. When train 1 was reproduced in an SA1 afferent fiber, its target WDR DHC also responded with a high degree of synaptic security, with each presentation evoking at least one spike in the dorsal horn neuron (mean $=2.0 \pm 0.6$ ) (Fig. $7 B$ ). However, in contrast to the response of the previous pair, both the first and second pairs of spikes in the input train raised the probability of AP generation in the postsynaptic neuron. These differences match those observed with CDPs in response to the same patterns of stimulation of an SAl and hair follicle afferent (Fig. 3, first and third traces).

The temporal characteristics of firing probability for the lowthreshold-only (LT) dorsal horn neuron displayed in Figure $8 A$, in response to stimulation of a hair follicle afferent with train 1, was almost identical to that seen for the hair afferent fiberWDR dorsal horn neuron pair (Fig. $7 A$ ), and the synaptic security was again $100 \%$. However, each stimulus presentation in this case evoked fewer spikes on average $(2.4 \pm 0.7)$. Therefore, although the amplitude of the response was different, the period of increased probability of discharge was the same (i.e., only the first pair of pulses evoked a response). When train 2 was delivered to the same afferent fiber, the synaptic security was slightly less $(92 \%$ of presentations evoked at least one spike; mean $1.3 \pm 0.8$ ) but the dorsal horn neuron again responded almost solely to the onset of the spike train (Fig. $8 \mathrm{~B}$ ). Contrasting results were observed when the stimulated afferent was an SAl. The paired LT dorsal horn neuron (different from the one in Fig. 8) responded to both the first and second pairs of pulses in train 1 and perhaps even slightly to the third pair (mean number of responses for the entire train $=2.7 \pm 0.9$ ) (Fig. 9A). When train 2 was presented to the same pair, the synaptic security dropped from $100 \%$ to $94 \%$ and the mean number of spikes per trial dropped to $2.2 \pm 1.0$. However, once again in contrast to the hair afferent-dorsal horn neuron pair, each component of the input spike train significantly raised the probability of spike generation in the dorsal horn neuron (Fig. 9B). Since the average response per trial consisted of only 2.2 spikes, the dorsal horn neuron obviously did not respond to all components during cach stimulus presentation.

\section{Intracellular recordings}

Although variations in the probability of firing of single spinal neurons were in agreement with the CDP data for hair follicle and SA1 afferent fiber stimulation, these data did not permit evaluation of whether the CDP and its modulation reflect spike or subthreshold activity. On a few occasions, it was possible to penetrate the target DHC and study the EPSPs directly. In Figure 10 , the SA1 afferent fiber from the pair shown in Figure 9 was activated using three stimulus paradigms while intracellularly recording the evoked response in a WDR dorsal horn neuron. The top trace is the averaged EPSP $(n=800)$ evoked in response to continuous $18 \mathrm{~Hz}$ stimulation of the fiber. As predicted from the SAl-CDP findings (Fig. $6 \mathrm{~A}$ of Koerber and Mendell, 1988), all polysynaptic EPSPs appeared to be suppressed at this stimulus frequency, leaving a relatively uncontaminated monosynaptic EPSP. Train 1 (middle trace; $n=52$ ) and train 2 (bottom trace; $n=17$ ) evoked EPSPs whose amplitude modulation was strikingly similar to that of SA1 afferent fiber-evoked CDPs (Figs. 3, 5).

In both of the two afferent fiber-dorsal horn neuron pairs shown in Figures 11 and 12, the afferent fiber innervated hair follicles. The first pair (Fig. 11) is the same as that whose extracellularly recorded activity was displayed in Figure 8. As shown earlier, this connection exhibited strong synaptic security. This strength of coupling was not confined to the monosynaptic input, but it was also evident in the finding that an apparent disynaptic EPSP was consistently present even during $18 \mathrm{~Hz}$ stimulation (Fig. 11, top trace; $n=445$ ). This tendency for hair follicle afferent-driven networks to exhibit synaptic security in polysynaptic components was noted previously (Fig. 6.4 in Koerber and Mendell, 1988).

At the slower repetition rates $(0.5 \mathrm{~Hz})$, stimulation of the fiber with train 1 was always successful in evoking spikes in the dorsal horn neuron, and so it was not possible to average the evoked EPSPs accurately. However, a single presentation of train 1 


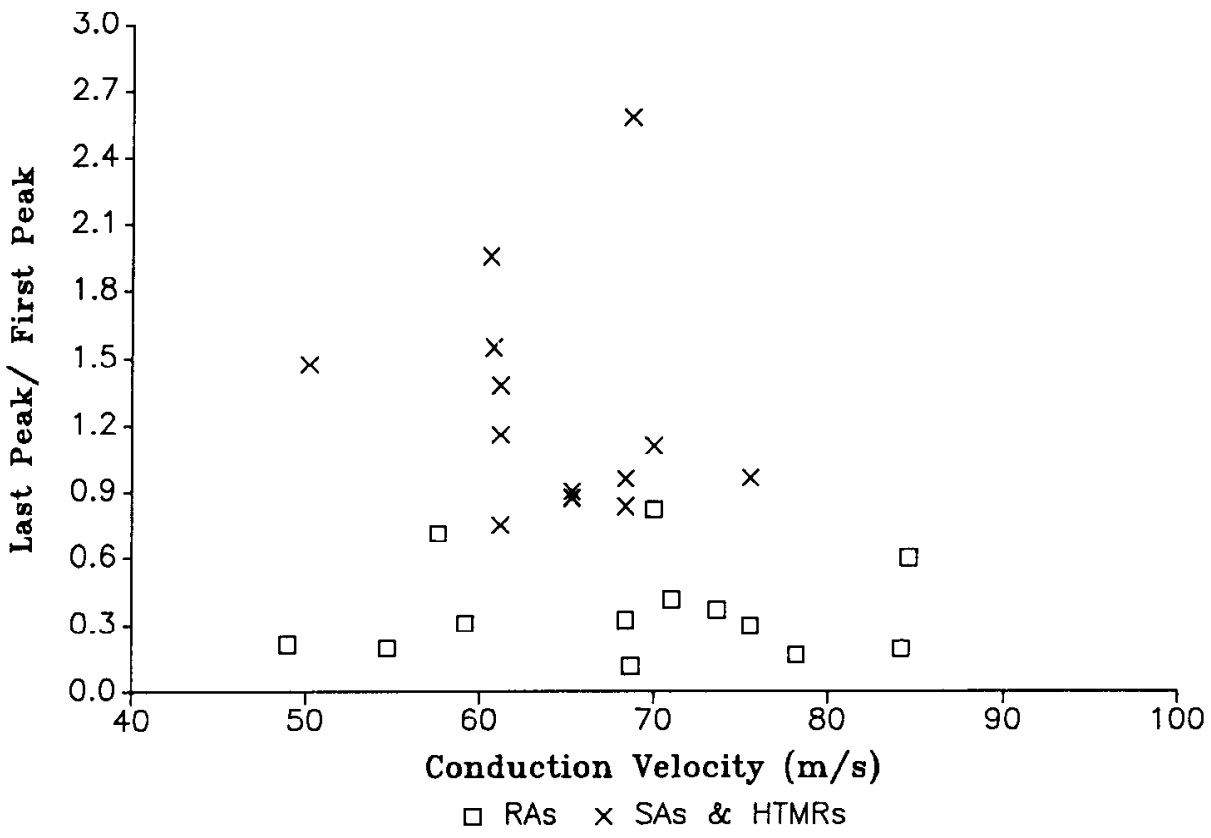

Figure 6. Differences in amplitude modulation of CDPs in response to train 2 as a function of peripheral receptor type. Ratio of the amplitude of the summed response to the last three stimuli to the amplitude of the response to the initial stimulus is plotted as a function of conduction velocity of the afferent. Note that the ratio is consistently higher for afferents innervating $S \Lambda$ receptors than for those innervating RA afferents and that this is independent of afferent conduction velocity. See further discussion in the text. elicited a burst of spikes in response to the first pair of impulses, and in this case a single spike was evoked in response to the second pair (Fig. 11, bottom trace). The third pair of afferent impulses evoked only subthreshold EPSP complexes.

The receptive field of the afferent in the second coupled pair was located at the edge of the postsynaptic neuron's receptive field (Fig. 12), in contrast to the first coupled pair (Fig. 11) in which the afferent's receptive field was close to the center. Stimulation of this afferent with train 1 did not appear to increase the probability of firing for this spinal neuron significantly (not shown), suggesting that they were not synaptically coupled. However, after penetrating it, it became apparent that the two cells were coupled. Although continuous stimulation of the afferent at $18 \mathrm{~Hz}$ did not evoke a visible monosynaptic response (Fig. 12, top trace; $n=500$ ), slower rates of patterned stimulation (train 1) revealed substantial polysynaptic linkage. The averaged EPSP complex (Fig. 12, bottom trace; $n=36$ ) had an onset latency of $2.5 \mathrm{msec}$, which after subtracting conduction time from the DRG was still greater than monosynaptic delay range. Although the strength of synaptic coupling differs from that of the previous pair, it is important to note that the dynamic behavior of the afferent fiber-dorsal horn neuron pair is similar (i.e., the first pair of pulses evoked a large response but the successive components evoked little or no response).

The results of these experiments with synaptically coupled pairs support the notion that the amplitude modulation of single fiber-croked CDPs reflects the amplitude modulation of single fiber-evoked EPSPs in individual DHCs.

\section{Discussion}

The present results indicate that differences in sensory coding of cutaneous information in the dorsal horn involve mechanisms beyond those that differentiate primary afferent neurons according to impulse encoding properties. The central projections of these fibers differ systematically in accordance with receptor type, implying that the relationship between firing of peripheral and central neurons will differ substantially in channels mediating input from different receptor types.
Our use of naturally occurring discharge patterns adds a degree of realism to the study of sensory processing in the sense that these are intervals that spinal networks would normally process. It is true that we have activated hair afferents with patterns derived from SAl afferents and vice versa, as well as with their own patterns. However, we attach no significance to the receptor type from which the trains were derived. Indeed, some components of the frequency patterns from the two afferents are similar, and our findings suggest that transmission is dependent on differences in receptor type of the stimulated afferent to a greater extent than differences in impulse patterns. Specifically, both the SA1 and hair follicle discharge patterns revealed that networks activated by SAl and HTMR afferents tend to reproduce each component of the discharge faithfully, whereas those activated by RA receptors tend to amplify a specific portion of the input (e.g., the onset for RA pads). It remains to be seen whether these networks would function in the same manner when subjected to wider ranges of patterned input and to the normal barrage of inputs over multiple fibers characteristic of natural stimulation. Nonetheless, the present findings are indicative of the functional complexity of the projections of different afferent types to the dorsal horn.

\section{Interpretation of cord dorsum potentials}

In order to employ single fiber-evoked CDPs as a measure of dorsal horn network function, we must first establish the relationship between CDP amplitude modulation and DHC activity. In earlier studies that examined evoked CDPs, it was concluded that the negative waves of the CDP are extracellular reflections of the stimulus-evoked EPSPs generated in the postsynaptic DHCs in response to electrical stimulation of peripheral nerves (reviewed in Hubbard et al., 1969). In addition, we have established that differences in single fiber-evoked monosynaptic CDPs accurately reflect anatomical differences (i.e., longitudinal extent and total numbers of boutons) in the central projections of single afferent fibers (Koerber et al., 1990).

The time course of CDPs recorded from the surface of the spinal cord in response to afferent stimulation at $18 \mathrm{~Hz}$ strongly 
A

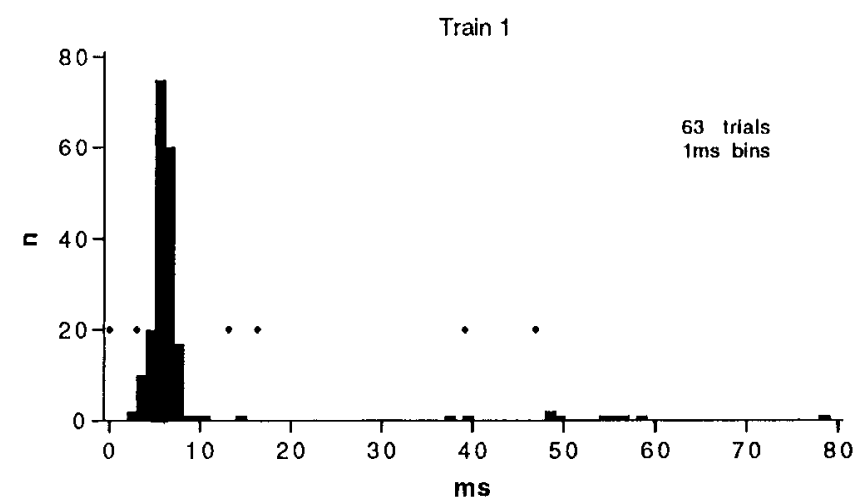

B

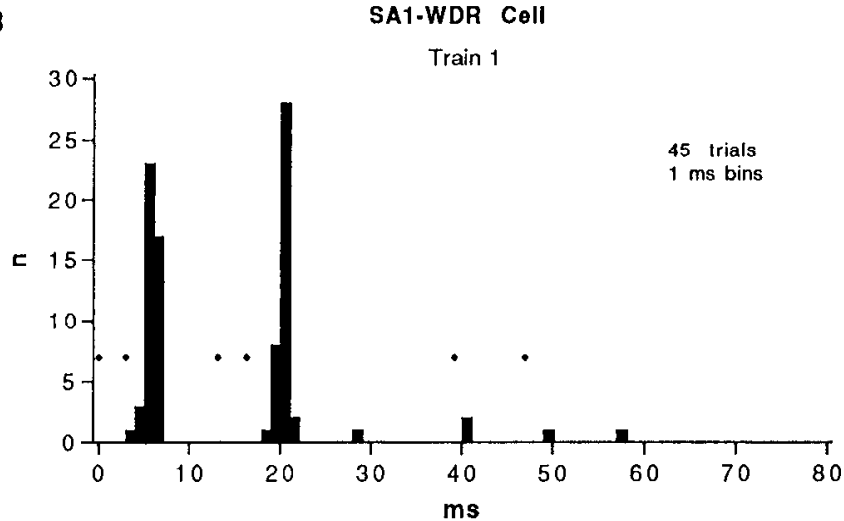

Figure 7. Response of a WDR neuron to stimulation of a hair afferent (A) and of a different WDR neuron to stimulation of an SAl afferent $(B)$ with train 1 . Stimuli are denoted by diamonds. The ordinate denotes the number of spikes occurring in each $1 \mathrm{msec}$ bin of the response over all trials. The hair afferent evokes a response only to the initial pair of APs in the afferent. In contrast, the SAl afferent evoked spikes in response to the first two pairs of APs. See further discussion in text.

suggested that for the most part these potentials reflect monosynaptic EPSPs produced in target dorsal horn neurons activated by the single fiber (Koerber and Mendell, 1988; Koerber et al., 1990). However, the CDPs produced by the trains of stimuli used here are more complex, due in part to the high intraburst frequencies, and also because of the long interburst intervals that would favor polysynaptic activity (Koerber and Mendell, 1988). Therefore, we have sought to determine to what extent the amplitude-modulated CDPs elicited under the conditions of these experiments provide a measure of the activity of individual spinal elements activated by the single fiber.

The results using synaptically coupled afferent fiber-DHC pairs clearly demonstrated, at least for the limited sample reported, that individual cells in the dorsal horn respond to individual hair afferent input only during the onset of the stimulus train, while they are capable of responding to most components of the input train delivered via SAl input channels, exactly as expected from the CDP results. In addition, those pairs in which the postsynaptic cell was also impaled revealed that modulation of single fiber-evoked EPSP amplitude is very similar to that of CDPs evoked by identical stimulation of the same afferent type. This was true even in cases where the input was subthreshold for the cell being studied (Figs. 10, 12).

Further support for this interpretation of modulation of CDP amplitude can be found in the earlier studies. Tapper et al.
A

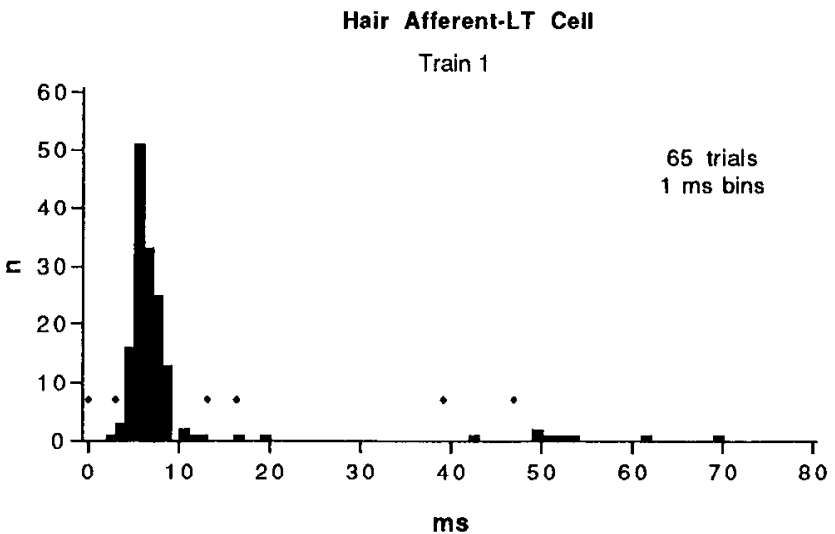

B

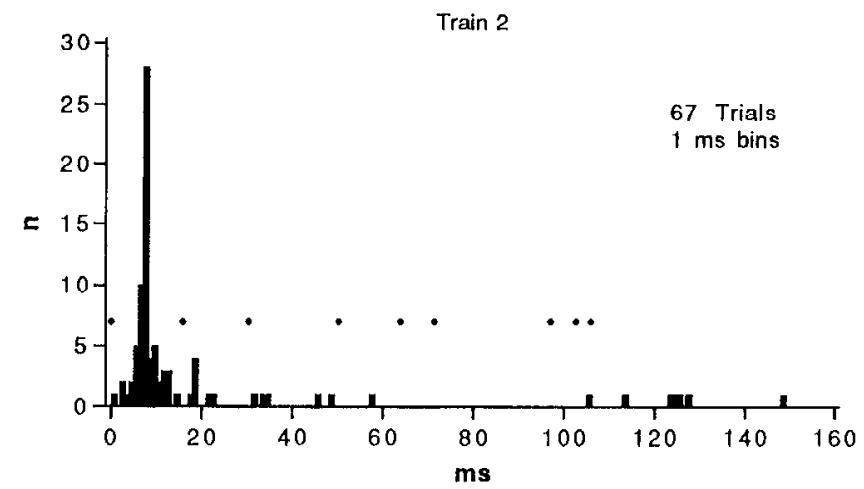

Figure 8. Response of an LT dorsal horn neuron to stimulation of a single hair follicle afferent. The ordinate is the same as in Figure 7. A Stimulation with train 1 (stimuli denoted by diamonds). Note that only the initial pair of stimuli evoked a substantial response. $B$, Response of the same afferent/neuron pair to stimulation with train 2 . Again, only an early response was noted. See further discussion in text.

(1983) reported that when pairs of pulses (interstimulus interval, $50 \mathrm{msec}$ ) were delivered to single SAl fibers, the conditioning stimulus had mixed effects on the response of the dorsal horn neuron to the test stimulus. In contrast, Brown et al. $(1987 \mathrm{~b}, \mathrm{c})$ found that similar pairs of stimuli delivered to hair follicle afferents always resulted in substantial depression of the response of spinocervical tract (SCT) cells to the test stimulus. Our previous study of single fiber-evoked CDPs was in complete agreement with these findings; that is, a conditioning pulse always reduced the hair afferent network's response to the test pulse, in contrast to the mixed effects observed for different SAI afferent-driven networks (Koerber and Mendell, 1988).

It is important to recall that CDPs reflect the average network response and not necessarily the activity of each of the individual network components. For example, Tapper and colleagues (Tapper and Wiesenfeld, 1980; Tapper et al., 1983, 1985) observed that individual neurons could respond to single APs elicited at low frequencies in SA 1 afferents with either an early response only, an early and a late response, or a late response only. Stimulation of hair follicle afferents elicited the same range of responses in SCT cells (Brown et al., 1987a-c). Thus, although the differences across afferent types are robust on the average, there may be considerable variation from afferent to afferent within a given type, reflecting perhaps the fact that afferents whose receptive fields are in the center of the receptive field of a postsynaptic cell tend to activate that cell much more strongly than afferents innervating the periphery of the central cell's re- 
A

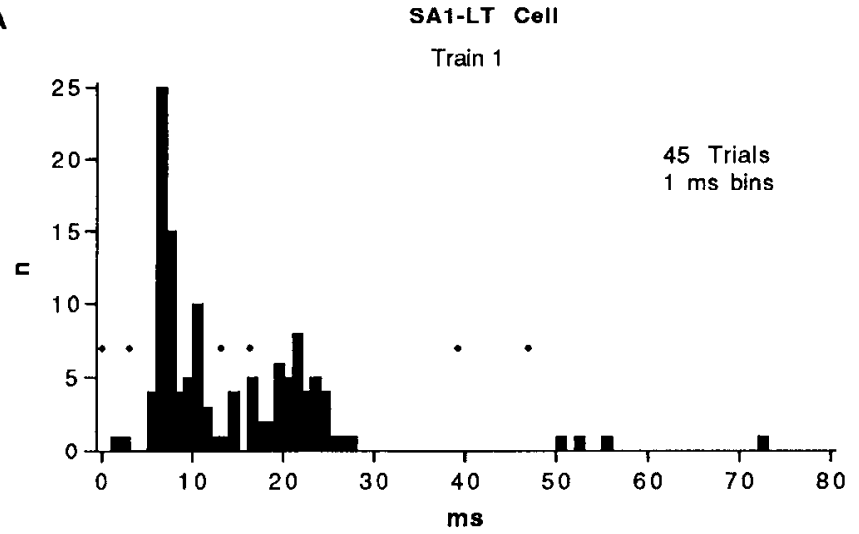

B

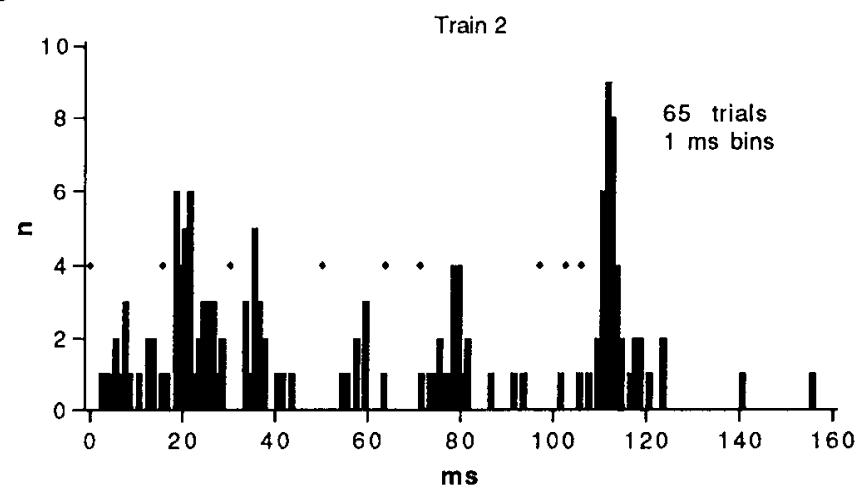

Figure 9. Response of an LT dorsal horn neuron to stimulation of an SAl afferent with train $1(A)$ and train $2(B)$. The ordinate is the same as in Figure 7 . Note the much more sustained responses compared to those evoked by stimulation of hair afferents with the same trains (Fig. 8). See further discussion in text.

ceptive field (Brown et al., 1987a-c; see also Brown and Noble, 1982). Another consideration is that not all cells in a network will contribute equally to generation of the CDP due to differences in depth, dendritic orientation, amplitude of synaptic potentials, etc.

\section{CDP amplitude modulation: mechanisms and functional implications}

In recent years, it has become apparent that the spinal dorsal horn is not merely a relay point for peripheral sensory information. Significant processing of inputs takes place at this level, so that signals transmitted to other spinal areas and higher centers can bear little resemblance to the input. The amplitude modulation of single fiber-evoked CDPs reported here demonstrates some aspects of this processing.

Although most of the afferent fiber-driven networks exhibited some sensitivity to the instantaneous frequency at the onset of afferent activity, they displayed clearly different response properties during the frequency-modulated burst, depending on the type of peripheral receptor innervated. For example, networks activated by hair follicle, field, and RA-pad afferent fibers responded primarily at the onset of afferent activity. Those driven by SA1, SA2, and HTMR afferent fibers responded throughout the discharge (e.g., compare response of dorsal horn neurons in Figs. $8 B, 9 B$ ). However, it is important to note that previous activity does affect the SA afferent fiber-driven networks to
SA1 - WDR CELL
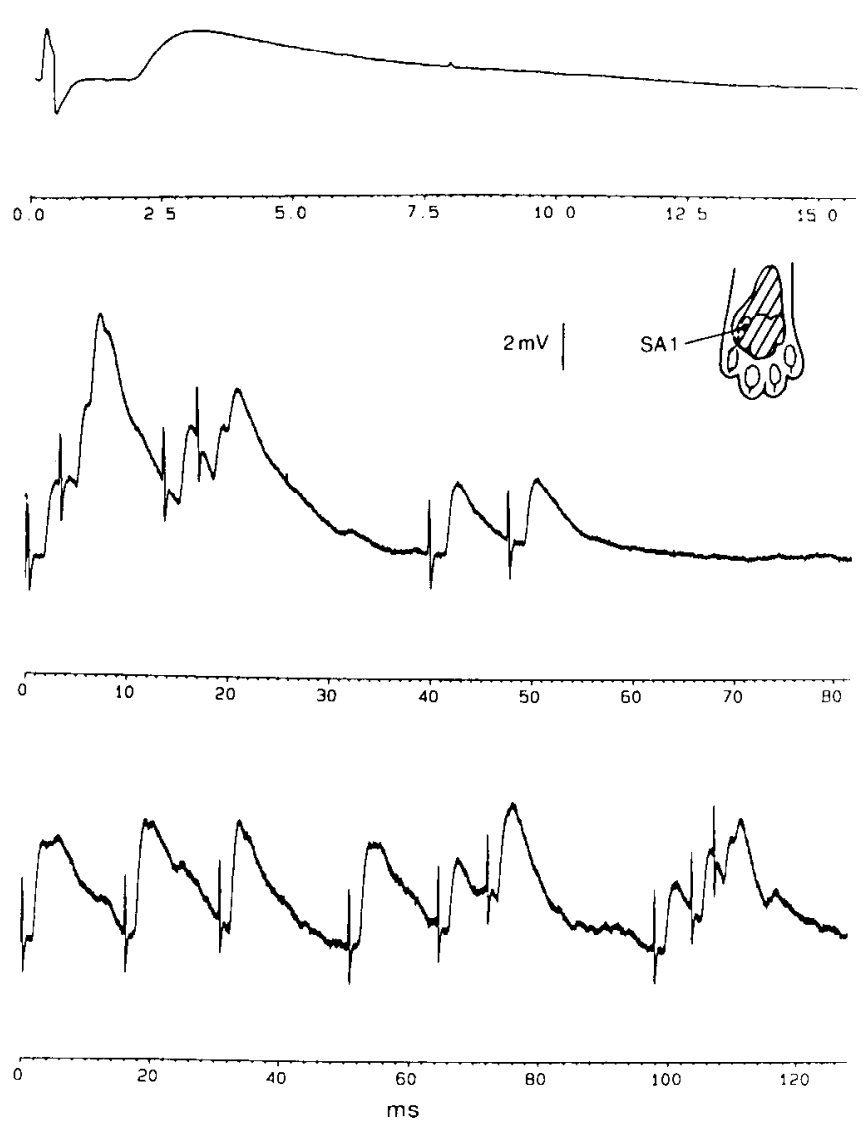

Figure 10. Averaged intracellular response of WDR cell to SA I afferent stimulation with three programs of stimulation $(n=$ number of responses averaged). Top, stimulation at $18 \mathrm{~Hz}(n=800)$. Middle, train $1(n=52)$. Bottom, train $2(n=17)$. Stimulus artifacts denote timing of the patterned input. Receptive field of dorsal horn neuron is shown as a hatched area (inset), with the much smaller receptive field of the SAl afferent near its edge. Note the similarity of the amplitude modulation of the EPSPs to modulation of the CDPs evoked by the same programs of SA1 stimulation displayed in Figure 2. See further discussion in text.

some degree. Although the interval between the first pair of spikes in train 1 (Fig. 3) is the same as that between the last two spikes in train 2 (Fig. 5), this pair of spikes in the same SA1 afferent evoked a larger network response when it appeared at the onset of the burst.

At present, we cannot specify how the networks activated by SA and RA afferents differ in their ability to process patterned input. One clue may come from previous work using pairs of impulses separated by $50 \mathrm{msec}$ delivered to SA and RA afferents (Koerber and Mendell, 1988). More sensitive hair follicle afferents tended to produce the largest polysynaptic CDP components, and test CDPs elicited by these afferents were the most susceptible to depression as a result of the conditioning stimulus. SAl afferent responses were largely monosynaptic, and these were the least sensitive to depression. In the present cxpcriments, single-unit recording, both extracellular and intracellular, confirmed the tendency of single impulses in hair afferents to evoke multisynaptic discharges, and this may permit cells activated by them to respond vigorously at the onset of a train 


\section{HAIR AFFERENT - LT CELL}
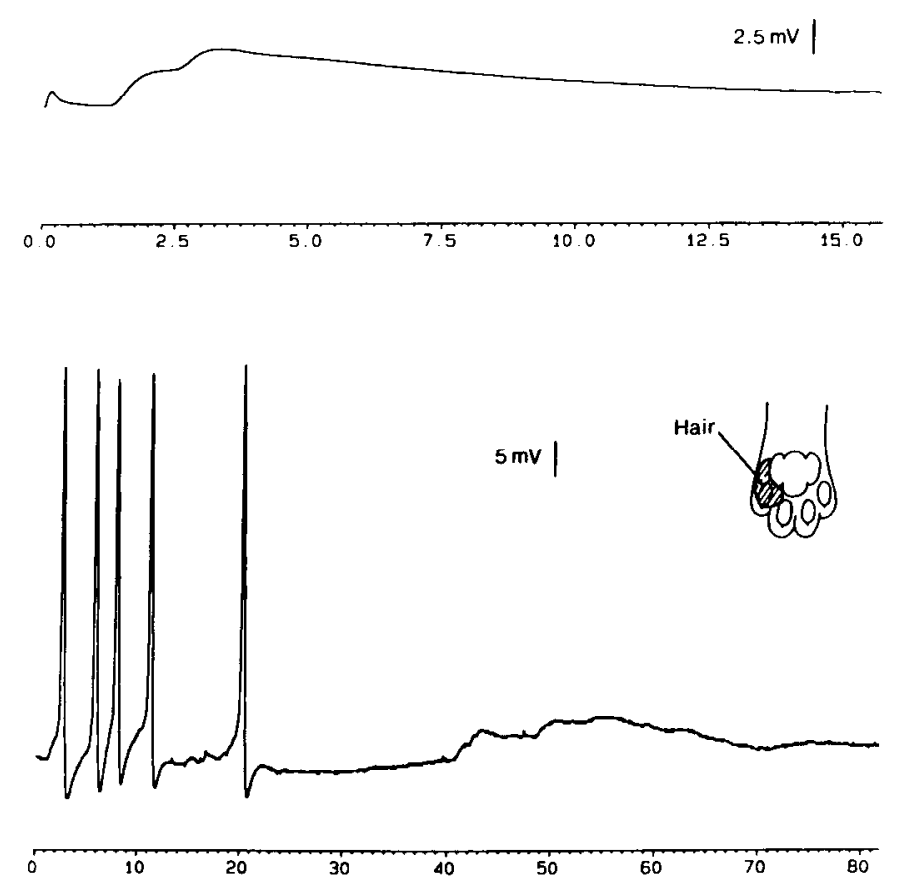

ms

Figure 11. Intracellular response of LT dorsal horn neuron to stimulation of a hair follicle afferent with $18 \mathrm{~Hz}$ stimulation (top: average, $n=445$ ) and to train 1 (bottom, response to single train presentation with timing the same as in middle trace of Fig. 10). Receptive fields of afferent and spinal neuron are shown in the inset. See further discussion in text.

but to be unable to maintain a discharge. These networks driven by individual hair afferents would be ideal for detecting the onset of a stimulus (e.g., movement of a hair) but would be less able to signal a steady stimulus. In contrast, SA 1 -activated networks would respond throughout a stimulus train but would not exhibit the large amplification of the initial response as seen for hair-activated networks.

Networks supplied by PC and D-hair afferent fibers, like those driven by other RA afferents, respond to high-frequency input by exhibiting an initial facilitation followed by depression. However, these networks appear to have a higher threshold of activation in that it takes a greater number of afferent spikes at high frequency to activate them fully. One possible reason for this different threshold level could be that many DHCs (e.g., $\mathrm{SCT}$ cells) receive a mixed excitatory/inhibitory input from these afferents, in contrast to an exclusively excitatory input from $\mathrm{A}_{3 j}$ hair follicle afferents (Brown et al., 1987c).

A recent study of SCT cells by Noble and Short (1989) suggests that the temporal interactions of the sort described here for RA afferents may also have a spatial component. Using brief puffs of air to activate small numbers of hair follicle afferents, they found that activation of afferents from almost any location in an SCT cell's receptive field would result in reduction of the response to the second stimulus even if the hairs activated by the two air puffs were separated by several centimeters. In addition, they found that stimulation of afferents that elicited only a subthreshold excitatory response was also capable of reducing the cell's response to a second stimulus.

\section{HAIR AFFERENT - WDR CELL}
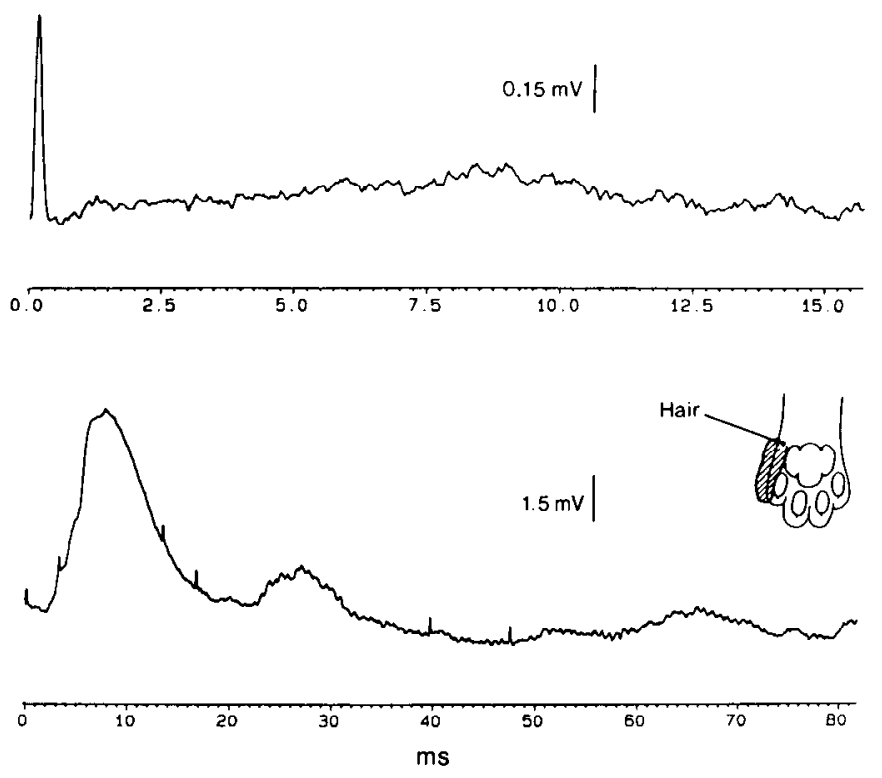

Figure 12. Averaged intracellular response of a WDR dorsal horn neuron to different programs of stimulation of a hair afferent. Top, Virtual lack of response to single APs $(n=500)$. Intracellular positioning of electrode was verified by steady negative DC membrane potential throughout response period. Bottom, Response to stimulation with train $1(n=36)$. Stimulus artifacts denote timing of afferent activation. Receptive fields are shown in inset. See further discussion in text.

These spatial interactions help to explain the significance of the different networks associated with fibers innervating RA receptors. In the absence of these interactions, one might postulate simply that networks responding mainly to the onset of activity accentuate the adaptation that takes place at the peripheral receptor. However, the spatial interaction means that this filtering is also organized at the level of the receptive field of the postsynaptic cell. Networks receiving inputs from RA afferents would tend to diminish the synaptic efficacy of other such afferents in the cell's receptive field. In effect, a DHC activated by RA afferents may have two states, one with a large receptive field capable of detecting the presence of an initial stimulus, and a second state where in the case of a more sustained stimulation, the receptive field shrinks to encompass only the more powerful inputs from the central region. This would increase spatial acuity, thus facilitating stimulus localization (see also Noble and Short, 1989).

These filtering characteristics also suggest possible mechanisms for the processing of temporal information. For example, it has been suggested that since hair follicle afferents can follow very high stimulus frequencies, they could serve as vibration detectors in hairy skin (Merzenich and Harrington, 1969). The adaptation observed for hair afferent-driven networks would at first seem to be at odds with this notion. However, the filtering out of the highly variable (both in amplitude and duration) lowfrequency (i.e., polysynaptic) EPSP components would be essential for the entrainment of postsynaptic cells to multiple synchronous high-frequency inputs. Therefore, these response characteristics of RA afferent-driven networks could serve to increase the temporal "acuity," that is, the ability of the network to encode high-frequency information. 


\section{Conclusions}

In summary, modulation of single-fiber CDP amplitude in response to frequency-modulated afferent activity varies according to the type of input channel (i.e., afferent/receptor complex) stimulated. Tapper et al. (1985) have suggested that single fiberdriven dorsal horn neuron networks are organized such that the identities of the input fibers (i.e., SA or RA) are "conserved and reflected in the output." A similar conclusion was reached in our recent study of CDPs employing conditioning-testing pairs of afferent pulses (Koerber and Mendell, 1988). The present study adds to these previous ones by employing physiologically realistic frequency-modulated trains of afferent pulses and by stimulation of additional classes of sensory fibers. The results of this study suggest that this generalization of network function may not apply to all types of afferent-driven networks. Regardless, the tuning implied by these receptor-specific transformations requires careful consideration in gaining an understanding of neural coding in cutaneous pathways.

\section{References}

Brown AG, Noble $R$ (1982) Connexions between hair follicle afferent fibres and spinocervical tract neurones in the cat: the synthesis of receptive fields. J Physiol (Lond) 323:77-91.

Brown AG, Koerber HR, Noble R (1987a) Excitatory actions of single impulses in single hair follicle afferent fibers on spinocervical tract neurones in the cat. J Physiol (Lond) 382:291-312.

Brown AG, Koerber HR, Noble R (1987b) Actions of trains and pairs of impulses from single primary afferent fibres on single spinocervical tract cells in the cat. J Physiol (Lond) 382:313-329.

Brown AG, Koerber HR, Noble R (1987c) An intracellular study of spinocervical tract cell responses to natural stimuli and single hair afferent fibres in cats. J Physiol (Lond) 382:331-354.

Brown PB, Moraff H, Tapper DN (1973) Functional organization of cat dorsal horn: spontaneous activity and central cell response to singlc impulses in single type I fibers. J Neurophysiol 36:827-839.

Collins WF III, Honig MG, Mendell LM (1984) Heterogeneity of group Ia synapses on homonymous alpha motoneurons as revealed by high frequency stimulation of Ia afferent fibers. J Neurophysiol 52:980-993.

Collins WF III, Davis BM, Mendell LM (1986) Amplitude modulation of EPSPs in motoneurons in response to frequency modulated trains in single la afferent fibers. J Neurosci 6:1463-1468.

Craig AD, Tapper DN (1985) A dorsal spinal neural network in cat. III. Dynamic nonlinear analysis of responses to random stimulation of single type 1 cutaneous input fibers. J Neurophysiol 53:995-1015.

Horch KW, Tuckett RD, Burgess RP (1977) A key to the classification of cutaneous mechanoreceptors. J Invest Dermatol 69:75-82.

Hubbard JI, Llinas R, Quastel DMJ (1969) Electrophysiological analysis of synaptic transmission. Baltimore: Williams and Wilkins.

Koerber HR, Mendell LM (1988) Functional specialization of central projections from identified primary afferent fibers. J Neurophysiol 60:1597-1614.

Koerber HR, Mendell LM (1991) Modulation of synaptic transmission at Ia afferent connections on motoneurons during high frequency afferent stimulation: dependence on motor task. J Neurophysiol 65: 1313-1320.

Koerber HR, Seymour AW, Mendell LM (1989) Tuning of spinal networks to temporal characteristics of spike trains in single primary afferents. Soc Neurosci Abstr 15:757.

Koerber HR, Brown PB, Mendell LM (1990) Correlation of monosynaptic field potentials evoked by single action potentials in single primary afferent axons and their bouton distributions in the dorsal horn. J Comp Neurol 294:133-144.

Mendell LM (1966) Physiological properties of unmyelinated fiber projection to the spinal cord. Exp Neurol 16:316-322.

Mcrzenich MM, Harrington T (1969) The sense of flutter-vibration evoked by stimulation of the hairy skin of primates: comparisons of human sensory capacity with the responses of mechanoreceptor afferents innervating the hairy skin of monkeys. Exp Brain Res 9:236260.

Noble R, Short AD (1989) Spatial spread of in-field afferent inhibition in the cat's spinocervical tract. J Physiol (Lond) 413:107-118.

Tapper DN, Wiesenfeld Z (1980) A dorsal spinal neural network in cat. I. Responses to single impulses in single type 1 cutaneous fibers. J Neurophysiol 44:1190-1213.

Tapper DN, Wiesenfeld Z, Craig AD Jr (1983) A dorsal spinal network in cat. II. Changes in responsiveness initiated by single conditioning impulses in single type 1 cutaneous input fibers. J Neurophysiol 49: 534-547.

Tapper DN, Craig AD, Brown PB (1985) Information transfer across a dorsal spinal neural network of cat in response to SAl and G-fiber input. In: Development, organization, and processing in somatosensory pathways (Rowe M, Willis WD, eds), pp 131-140. New York: Liss.

Willis WD, Coggeshall RE (1978) Sensory mechanisms of the spinal cord. New York: Plenum. 